\title{
A Temperature Sensor Implant for Active Implantable Medical Devices for In Vivo Subacute Heating Tests Under MRI
}

\author{
Berk Silemek (1D, ${ }^{1}$ Volkan Acikel, ${ }^{2}$ Cagdas Oto, ${ }^{3}$ Akbar Alipour (1D, ${ }^{1,4}$ \\ Zaliha Gamze Aykut (D), ${ }^{5}$ Oktay Algin, ${ }^{1,6}$ and Ergin Atalar ${ }^{1,4 *}$
}

Purpose: To introduce a temperature sensor implant (TSI) that mimics an active implantable medical device (AIMD) for animal testing of MRI heating. Computer simulations and phantom experiments poorly represent potential temperature increases. Animal experiments could be a better model, but heating experiments conducted immediately after the surgery suffer from alterations of the thermoregulatory and tissue properties during acute testing conditions. Therefore, the aim of this study was to introduce a temperature sensor implant that mimics an AIMD and capable of measuring the electrode temperature after implantation of the device without any further intervention at any time after the surgery in an animal model. Methods: A battery-operated TSI, which resembled an AIMD, was used to measure the lead temperature and impedance and the case temperature. The measured values were transmitted to an external computer via a low-power Bluetooth communication protocol. In addition to validation experiments on the phantom, a sheep experiment was conducted to test the feasibility of the system in subacute conditions.

Results: The measurements had a maximum of $0.5^{\circ} \mathrm{C}$ difference compared to fiber-optic temperature probes. In vivo animal experiments demonstrated feasibility of the system.

Conclusion: An active implant, which can measure its own temperature, was proposed to investigate implant heating during MRI examinations. Magn Reson Med 79:2824-2832, 2018. () 2017 International Society for Magnetic Resonance in Medicine.

Key words: implant heating; active implantable medical devices; in vivo heating; heating reduction; parallel transmission; Internet of Things

\footnotetext{
${ }^{1}$ National Magnetic Resonance Research Center (UMRAM), Bilkent University, Ankara, Turkey.

${ }^{2}$ Aselsan, REHIS Power Amplifier Technologies, Ankara, Turkey.

${ }^{3}$ Department of Anatomy, Ankara University, Ankara, Turkey.

${ }^{4}$ Department of Electrical and Electronics Engineering, Bilkent University, Ankara, Turkey.

${ }^{5}$ Department of Molecular Biology and Genetics, Bilkent University, Ankara, Turkey.

${ }^{6}$ Department of Radiology, Ataturk Training and Research Hospital, Ankara, Turkey.

Grant sponsor: Turkish Ministry of Science, Industry, and Technology; Grant number: 0204.STZ.2013-1.

*Correspondence to: Ergin Atalar, Ph.D., National Magnetic Resonance Research Center (UMRAM) and Department of Electrical and Electronics Engineering, Bilkent University, 06800 Ankara, Turkey.

E-mail: ergin@ee.bilkent.edu.tr; Twitter: @erginatalar
}

Received 5 February 2017; revised 17 August 2017; accepted 17 August 2017

DOI 10.1002/mrm.26914

Published online 14 September 2017 in Wiley Online Library (wileyonlinelibrary.com).

(C) 2017 International Society for Magnetic Resonance in Medicine

\section{INTRODUCTION}

In this study, a temperature sensor implant (TSI) was introduced for subacute in vivo MRI heating experiments of active implantable medical devices (AIMDs) such as deep brain stimulators in animals. A TSI mimics the electrical behavior of an AIMD and reports the tip and case temperatures wirelessly in real time during an MRI experiment.

MRI examination of patients with an AIMD can be problematic, because the metallic parts of an implant may interact with the radiofrequency (RF) electromagnetic fields, which may cause excessive heating of the tissue around them. The lead of the implant is a long conductor that may act as an antenna inside the MRI scanner. The RF energy applied during imaging may produce a highly localized electric field around the case and/or the electrode of the lead; consequently, excessive temperature increases near the electrodes or the case of the implants may be observed (1). Recently, our group and others have developed techniques to reduce implant heating by steering electric fields away from the implant (2-8). These techniques have to be rigorously tested before use in clinical practice.

The RF interaction of implants during MRI has been shown with experiments, models, and simulations in vitro $(1,9)$. The experimental studies indicated that MRIinduced heating depends on many parameters. For example, the lead path and device position in the body affect the temperature increase at the tip (10-12). In addition, sequence parameters are important and can be summarized as applied specific absorption ratio (SAR) values (13-15). Furthermore, other studies have assessed the static field strength dependency on the implant heating $(10,16)$. Yeung et al. (17) modeled the heating problem with a safety index as the ratio of temperature increase per applied unit SAR value. Additionally, Acikel et al. $(18,19)$ used a transmission line approach to determine the relationship between RF fields and induced currents as a function of wire length, insulation thickness, and lead radius, as well as the electrical conductivity and permittivity of tissue. The transfer function method was used for heating problems with the tip of the electrode (20). There are also a number simulation studies that can predict similar results with phantom experiments $(21,22)$; however, phantoms are homogeneous and can only be used to simulate the mean electrical conductivity and permittivity values of human tissue. Additionally, perfusion and blood flow are 
difficult to model, although there were attempts to solve this issue (23). Therefore, in vivo experiments are needed to verify computer models and phantom studies.

There are several in vivo studies that have assessed the AIMD heating problem. For example, Gorny et al. (24) reported in vivo deep brain stimulator heating at both $1.5 \mathrm{~T}$ and $3.0 \mathrm{~T}$ in an acute pig experiment, and they recorded temperature increases of $2.3^{\circ} \mathrm{C}$ by using fiberoptic temperature probes. Akca et al. (23) performed temperature calculations and validations in in vivo acute experiments using fiber-optic temperature probes. In that study, the calculations underestimated temperature increases in vivo. One possible reason for this underestimation is the difference between the perfusion values in the literature and experimental ones. However, the thermal, electrical, and thermoregulatory properties of the tissue may be altered immediately after surgery, and the implant heating experiments conducted immediately after surgery may thus result in bias in the temperature measurements. Ideally, the animal heating experiments should be conducted after complete recovery from the surgery for more accurate results.

To our knowledge, there is only one report in the literature of in vivo animal experiments under chronic conditions. Luechinger et al. (25) proposed thermocouples as a temperature measurement tool to test heating around pacemaker lead screws and reported a temperature increase of $20^{\circ} \mathrm{C}$ in an in vivo chronic case in pigs. However, the long conductive cables used for temperature measurements may introduce additional heating. More importantly, long after implantation, it becomes questionable whether the temperature measurement is from the maximum heating location or not.

The goal of this study was to develop a system that enables investigation of MRI heating experiments as close as possible to the MRI examination of patients with AIMDs at any time after implantation surgery. For this purpose, we designed a TSI with embedded temperature sensors, an electrode impedance measurement sensor, and wireless communication capability and demonstrated that this device is capable of collecting experimental data without further intervention to the animal or the device.

\section{METHODS}

\section{Implant Design}

The TSI was designed to mimic a typical deep brain stimulator implant with regard to its shape, size, and materials. By doing so, we assumed that the electromagnetic scattering from the TSI is similar to the scattering from an implant with similar size and shape. The resulting temperature increase of the tissue around the electrode should also be similar. The case was titanium alloy with a circular shape of $50 \times 40 \times 10 \mathrm{~mm}$ thickness. It was coated with 2-mm-thick polydimethylsiloxane. An area of $150 \mathrm{~mm}^{2}$ on the case surface was remained uncoated for electrical contact with the tissue. The implant was designed to prevent any infections due to the incompatible materials; all components that touch the tissue were medical grade, which is explained in further detail in the following sections.
A 60 -cm-long lead with a diameter of $2.3 \mathrm{~mm}$ was connected to the case. The electrode at the tip of the lead was composed of $99.9 \%$ silver with a thickness of $10 \mu \mathrm{m}$ and a $6-\mathrm{mm}^{2}$ surface area. A thermocouple was placed inside the silver electrode. Ports of the thermocouple were connected to the TSI circuit via a twisted pair, which was elongated through the lead. A braided copper shield was placed along the lead that was electrically connected to the electrode. To increase the yield strength of the lead, polytetrafluoroethylene tubing $(\mathrm{r}=1.8 \mathrm{~mm})$ was placed between the twisted pair and the copper shield. On the upper layer of the shield, the lead was electrically isolated, except for the electrode, using a biocompatible heat shrink with $0.2-\mathrm{mm}$ wall thickness.

\section{Circuit design}

The schematic of the circuit is given in Figure 1. For data recording and transfer, a BLE112 Bluetooth 4.0 module (Bluegiga; Bluegiga Technologies, Espoo, Finland) was used. BLE112 has differential input analog to digital converters (ADC), general purpose input/output ports, and the ability to communicate via a Bluetooth low energy (BLE) wireless connection. BLE112 was supplied with a 3.6-V, $100 \mathrm{~mA} \cdot \mathrm{h}$ button cell lithium-ion battery (VARTA Microbattery GmbH, Ellwangen, Germany). ADC1 and ADC2, of the ADC channels, were used for temperature recording, and ADC3 was used for the electrode impedance measurement. ADC channels feature 12-bit resolution, a $132-\mu$ s conversion time, and $2.97-\mathrm{V}$ full-scale signal amplitude.

Three of the general purpose input/outputs $\left(\mathrm{V}_{\mathrm{et}}, \mathrm{V}_{\mathrm{ct}}\right.$, $\mathrm{V}_{\mathrm{imp}}$ ) were used to supply temperature and electrode impedance measurement modules. Temperature measurement modules used NTC thermistors $\left(\mathrm{R}\left[25^{\circ} \mathrm{C}\right]=10\right.$ $\mathrm{k} \Omega \pm 1 \%, \beta=3950 \mathrm{~K}$; Cantherm, Montreal, Quebec, Canada) as a thermocouple. The case and electrode temperature measurements were performed with two NTC thermistors connected to Wheatstone bridges with three $13.3 \mathrm{k} \Omega$ resistors. To conserve power, all measurements were made with pulsed signals. The case temperature pulse was applied from $\mathrm{V}_{\mathrm{ct}}$ upon request from the user, and the voltage between ADC1 + and ADC1 - was differentially recorded. Electrode temperature was also measured in a similar fashion with the case temperature. However, the thermistor was placed inside the electrode, and a connection was established via a $60-\mathrm{cm}$ twisted cable. Although the connection to the thermistor was a twisted pair cable inside of a copper shield, it was still prone to interference, as it works under high RF and gradient pulses. Hence, 1-nF capacitors between the thermistor connection and ground were placed to decrease interference. However, capacitors caused some delay when the pulse, $\mathrm{V}_{\text {et }}$, was applied. This problem was solved by adjusting the timing of the data acquisition of the ADC. With this setup, the electrode temperature was recoded every second. In addition, the impedance measurement module was implemented using a DC block capacitor and a current sensing resistor and connected to the electrode via the shield of the lead. To measure the electrode impedance, a pulse was applied from the port $\mathrm{V}_{\mathrm{imp}}$, and ADC3 was turned on. Similar to the case 


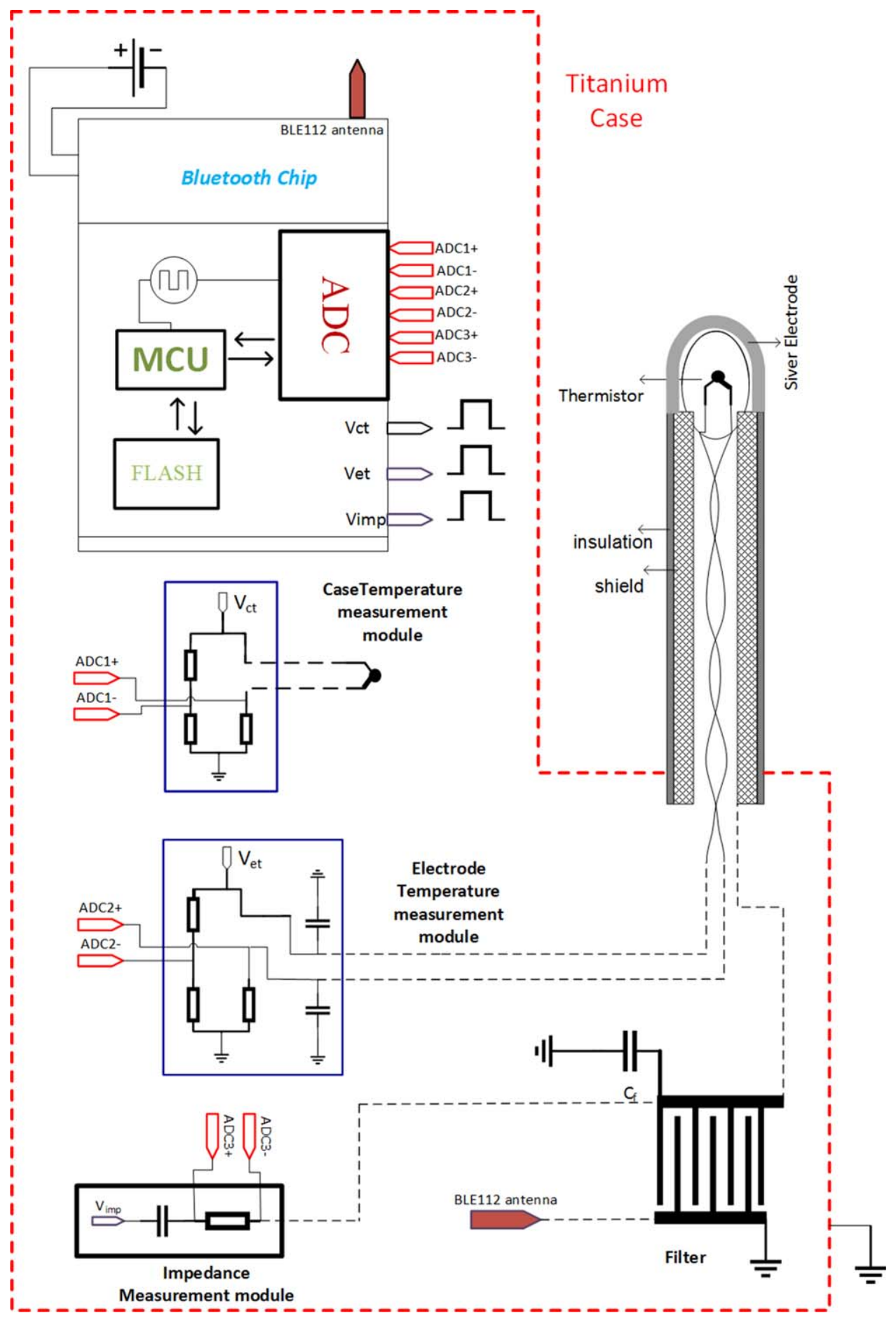

FIG. 1. TSI schematic diagram. The thermistor body and its connections are inside the silver electrode and the shield; therefore, the thermistor itself does not influence the RF-induced heating.

temperature, electrode impedance was also recorded by user request. All recorded data were transferred via Bluetooth to a personal computer (PC) or an android device.

Because the TSI circuitry was encapsulated in the titanium case, the on-chip Bluetooth antenna of the BLE112 was not operational. To establish communication between the target device and BLE112, an external antenna, which was connected to BLE112 on the titanium case, was needed. Hence, the shield of the lead was used as a Bluetooth monopole antenna where the ground plane was the titanium case. The risk of harming the antenna, $\mathrm{V}_{\mathrm{imp}}$, and the ADC3 port of the BLE112 due to induced currents on the shield during the MRI experiment was addressed by placing the microstrip Lange coupler as a filter (Fig. 1). Using this filter, induced current on the shield was directed to the titanium case via the $\mathrm{C}_{\mathrm{f}}$, and the Bluetooth signal was directed to the BLE112 antenna port. The duration of the $V_{\text {imp }}$ pulse and the ADC3 opening timing were adjusted such that the measurement was not effected by $\mathrm{C}_{\mathrm{f}}$. 


\section{Software design}

TSI has three software components: Bluetooth module software and computer and mobile applications. The Bluetooth module was programmed with the scripting language of the Bluetooth chip. The case temperature and electrode impedance were measured upon user request. The temperature measurement was performed every one second if it was enabled by the user.

Additionally, connection parameters such as communication intervals, slave latency, and implant advertisement parameters were made adjustable to save battery life. The battery voltage level can also be tracked wirelessly by the user, and it was dynamically updated in the software to determine voltage of the supply ports of the Wheatstone bridges and impedance measurement network. In addition, receiver signal strength indication (26) related to the connection of the TSI and a PC was measured by the software to detect range of the communication empirically under MRI environment.

During MRI experiments, the PC was placed at the outside of the scanner room, next to the scanner's computers. A 3-m-long USB extender cable was connected to the PC to place a Bluetooth dongle (BLED112, Bluegiga Technologies) near the scanner bore. A Java-based PC application was developed to use this system during the MRI experiment. However, using a PC was not practical to collect data when the animal was under surveillance or surgery. Hence, an android (version 4.3) mobile application was also developed. Both applications had the similar capabilities and graphical user interface.

\section{Experiments}

All MRI experiments were performed on a 3T MRI scanner (TimTrio, Siemens Medical Solutions, Erlangen, Germany) with an eight-channel transmit array capability. The body birdcage coil in dual-drive mode was used as the transmit coil. For this, the quadrature splitter of the system was removed and the two ports of the birdcage coil were fed using two channels of the eight-channel transmit array system. A 12-channel head coil and a body matrix coil (Siemens Medical Solutions, Erlangen, Germany) were used for animal experiments as receiver coils; only the body matrix coil was used for phantom experiments.

\section{Phantom experiments}

Once the TSI had been constructed, the system was verified with an infrared thermometer (ScanTemp-330; TFA Dostmann, Wertheim, Germany). We took temperature values between $20^{\circ} \mathrm{C}$ and $60^{\circ} \mathrm{C}$ from the TSI and infrared thermometer. Then, to validate TSI functionality in an MRI environment, a phantom (14 g/L hydroxyethylcellulose solution and $1.5 \mathrm{~g} / \mathrm{L} \mathrm{NaCl}$ ) experiment was performed. A rectangular phantom $(65 \times 40 \mathrm{~cm})$ was placed to be cocentered with the body birdcage coil. The TSI was placed inside the phantom, and a fiber-optic temperature sensor (sensor: $\mathrm{T}_{1}$, signal conditioner: REFLEX; Neoptix, Quebec City, Quebec, Canada) was placed as close as possible (no more than $1 \mathrm{~mm}$ away) to the electrode. The temperature around the lead was recorded using both TSI and the fiber-optic sensor during the MRI sequence for $15 \mathrm{~min}$. The case temperature was also measured with the TSI only at the start and at the 7 th minute of the MRI scan.

\section{Animal experiments}

Animal experiments were designed to illustrate a subacute in vivo application of the TSI and the in vivo effectiveness of the implant friendly mode of a dualdrive birdcage coil $(2,3)$. The experimental protocol was approved by the ethics committee for animal research of Bilkent University (Ankara, Turkey). We used an adult, nonpregnant, healthy female sheep weighing $45 \mathrm{~kg}$ that was collected from the Ankara University Faculty of Veterinary Medicine. All brain lead implantation procedures were performed in our animal surgery room with sterile conditions. Food and water were withheld for 24 and 6 hours before surgery, respectively. The animal was premedicated with $0.02 \mathrm{mg} / \mathrm{kg}$ atropine sulfate (atropine sulfate ampoule; Biofarma, Istanbul, Turkey) and $0.05 \mathrm{mg} / \mathrm{kg}$ xylazine $\mathrm{HCl}$ (Alfazyne $2 \%$; Egevet, Istanbul, Turkey). These drugs were administered intramuscularly approximately $15 \mathrm{~min}$ before the induction of anesthesia. Anesthesia was administered with $10 \mathrm{mg} / \mathrm{kg}$ ketamine $\mathrm{HCl}$ (Alfamine 10\%; Egevet) injected intramuscularly. The sheep was positioned in lateral recumbency. During the MRI scan, anesthesia was maintained with total intravenous anesthesia of propofol $10-14 \mathrm{mg} / \mathrm{kg} / \mathrm{h}$, $10 \mathrm{mg} / \mathrm{kg} / \mathrm{h}$ (Propofol; Fresenius, Frankfurt, Germany) in $0.9 \% \mathrm{NaCl}$ solution (Eczacibasi Baxter, Istanbul, Turkey). The sheep received continuous intravenous infusion of lactated Ringer's solution at a rate of $10 \mathrm{~mL} / \mathrm{kg} / \mathrm{h}$ (Eczacibasi Baxter) during anesthesia.

Postoperative analgesia consisted of a 3-day course of intravenous $1 \mathrm{mg} / \mathrm{kg}$ dexketoprofen trometamol (Arveles; Ufsa, Istanbul, Turkey) twice a day. Rifampicin (250 mg, Rifocin ampoule; Sanofi Aventis, Istanbul, Turkey) was administered subcutaneously to surgical sites after surgery. Dexamethasone, with the initial dose of $8 \mathrm{mg}$ (Dekort ampoule; Deva, Istanbul, Turkey), was injected intramuscularly for 4 days postoperatively by reducing the initial dose in half each day. In addition, $100 \mathrm{~mL}$ of $20 \%$ mannitol solution (Eczacibasi Baxter) was administered intravenously twice a day for 3 days, and $500 \mathrm{~mL}$ of $5 \%$ dextrose solution (Eczacibasi Baxter) and $500 \mathrm{~mL}$ of $0.9 \% \mathrm{NaCl}$ solution (Eczacibasi Baxter) were administered intravenously for 3 days postoperatively. Antibiotics were provided for 7-10 days postoperatively: $2 \mathrm{~g}$ of ceftriaxone (I.E. Ulagay, Iesef flakon) was injected intramuscularly twice a day, and $1 \mathrm{~g}$ of vancomycin (Vancotek flakon; Kocak Farma, Istanbul, Turkey) was administered as a continuous intravenous infusion (in $0.9 \% \mathrm{NaCl}$ solution) twice a day.

Before placement of the implant, MR images were acquired in the lateral and head-first position in the normal operation mode of the scanner for surgical planning. After acquisition of scout images, sagittal $3 \mathrm{D} \mathrm{T}_{1}$-weighted (3D-MPRAGE), $\quad \mathrm{T}_{1}$-weighted (3D-SPACE), and $\mathrm{T}_{2^{-}}$ weighted (3D-SPACE with variant flip angle mode) sequences were acquired with the isotropic voxel sizes and parameters in Table 1. All of the sequences covered the whole brain. The acquisition time of each MR 
Table 1

MR Examination Protocol of the Study

\begin{tabular}{|c|c|c|c|}
\hline Sequence Parameters & $\mathrm{T}_{1}$-Weighted 3D-MPRAGE & $\mathrm{T}_{1}$-Weighted 3D-SPACE & $\mathrm{T}_{2}$-Weighted 3D-SPACE \\
\hline Repetition time, ms & 2130 & 700 & 3000 \\
\hline Echo time, ms & 3.86 & 12 & 430 \\
\hline Field of view, mm & $120 \times 100$ & $210 \times 210$ & $174 \times 100$ \\
\hline No. of averages & 1 & 1 & 1 \\
\hline Slice thickness/separation, $\mathrm{mm}$ & $0.47 / 0$ & $0.82 / 0$ & $0.68 / 0$ \\
\hline Voxel size, $\mathrm{mm}$ & $0.47 \times 0.47 \times 0.47$ & $0.82 \times 0.82 \times 0.82$ & $0.68 \times 0.68 \times 0.68$ \\
\hline No. of slabs & 1 & 1 & 1 \\
\hline Flip angle & $12^{\circ}$ & Variant & Variant \\
\hline Inversion time, ms & 1100 & NA & NA \\
\hline No. of slices & 192 & 208 & 192 \\
\hline Phase oversampling, \% & 10 & 10 & 50 \\
\hline
\end{tabular}

Abbreviations: 3D-MPRAGE: 3D magnetization-prepared rapid gradient-echo; 3D-SPACE, 3D sampling perfection with applicationoptimized contrasts by using different flip angle evolutions; NA, not applicable.

examination was approximately $20 \mathrm{~min}$ for the evaluation of the targeted lead position. All 3D sequences were evaluated on a dedicated workstation (Leonardo; Siemens, Erlangen, Germany), and thin-slice reformatted images were obtained in multiple planes (axial, coronal, and oblique). Additionally, curved reformat, multiplanar reconstruction, fused, maximum intensity projection images were obtained with the workstation. All of the MRI images obtained were evaluated by an experienced neuroradiologist.

A $10-\mathrm{cm}$ skin incision at the right shoulder was performed to form a pocket. A tunnel was formed using the trocar of a standard size adult port catheter to carry the lead of the implant. The tip of the lead was placed on the frontal lobe white matter. After observing the functionality of the system and the position of the case, the incision sites were sutured, and the surgical procedure was completed.

Immediately after placement of the TSI, the animal was transferred to the scanner room to confirm the position of the lead. To avoid possible thermal damage to the brain of the animal at this stage of the experiment, imaging was conducted using the implant-friendly mode described by Eryaman et al. (3). In short, by using a very low flip angle (and therefore low power) gradient echo pulse sequence with a repetition time of $7 \mathrm{~ms}$, echo time of $3 \mathrm{~ms}$, and field of view of $200 \mathrm{~mm}$, the RF pulses were applied using the dual-drive birdcage coil with the RF signal at the same phase to both terminals of the coil. This produced a linearly polarized field. The direction of the magnetic field was rotated by adjusting the relative amplitudes of the voltages applied to the ports. At one angle, the E-field coupling to the TSI was at its maximum, and perpendicular to this angle, the coupling was at its minimum (implant friendly mode). In the case of the maximum coupling condition, the induced current on the wire caused increased in flip angle around the lead of the implant. This was apparent in the gradient echo images as signal intensity increase. The temperature of the implant electrode was monitored continuously to make sure that no significant temperature increase occurred during the procedure.

After the surgery, the sheep was maintained under observation in the animal shelter of our facility for healing. On the 14th day, the animal MR heating experiment was performed. For positioning, the scanner's landmark was closer to the implant tip. No other position changes were made during the scan. After finding the implantfriendly mode as described above, the anatomical images were obtained using the same imaging protocol with the parameters provided in Table 1. During imaging, the temperature increase was monitored with the TSI.

After anatomical imaging, the high whole body averaged SAR sequence was applied in the implant-friendly and maximum coupling modes, and the tip temperature was recorded with 1-s time resolution during the sequence. The impedance value and case temperature were measured before the scan, in the middle of the sequence, and after the sequence when the electrode temperature reached the base temperature value. Finally, the birdcage coil channels were driven with the same amplitude and $90^{\circ}$ phase difference as in the quadrature mode, and the heating experiment was repeated. For heating, rectangular RF pulses with a duration of $2.5 \mathrm{~ms}$ were applied with a repetition time of $7.5 \mathrm{~ms}$. The applied voltage to each coil port was $300 \mathrm{~V}$, which was scaled with cosine and sine to obtain the aforementioned implant-friendly and maximum coupling modes. Additionally, port reflections were measured using the builtin port reflection function of the Siemens service menu. Port reflections and scaling constants are given in Table 2 ; according to these values, the calculated whole body average SAR was $7 \mathrm{~W} / \mathrm{kg}$ for all modes of operations.

\section{RESULTS}

The Bluetooth antenna filter had an insertion loss of 0.9 $\mathrm{dB}$ at a 2.4-GHz Bluetooth frequency and $66 \mathrm{~dB}$ at 123 $\mathrm{MHz}$, the Siemens 3T MRI frequency. The Bluetooth communication range of the system had a maximum of $2 \mathrm{~m}$ in phantom and animal experiments. The advertisement interval adjusted to $10 \mathrm{~s}$ and resulted in a $10-\mu \mathrm{A}$ average current drain for no connection. The communication intervals were adjusted to $0.5 \mathrm{~s}$ with 1-cycle latency. This corresponded to $1 \mathrm{~Hz}$ data transmission rate with $26-\mu \mathrm{A}$ average current drain for MR experiment mode from the battery. The estimated battery life was approximately 13 months for no connection with 10-s advertisement intervals and 5 months for the MR experiment mode. The picture of the TSI is given in Figure 2. 
Table 2

Port Reflection Values of Birdcage I and Q Channels

\begin{tabular}{lcccc}
\hline & & \multicolumn{3}{c}{ Amplitude Scale Constants } \\
\cline { 3 - 5 } Channel & Port Reflection Values & Low E-Field Plane & High E-Field Plane & Quadrature Mode \\
\hline I & 0.76 & 0.64 & 0.77 & 0.71 \\
Q & 0.68 & 0.76 & 0.64 & 0.71 \\
\hline
\end{tabular}

The scalars are the amplitude scale of each birdcage port for each experiment.

The temperature measurements for both the case and the tip were verified with an infrared thermometer for the temperature ranges between $20^{\circ} \mathrm{C}$ and $60^{\circ} \mathrm{C}$ with the accuracy of $2^{\circ} \mathrm{C}$, which was the limit of the infrared thermometer. The system was further tested with a phantom experiment by comparing the fiber-optic probe and the TSI temperature measurements, which are shown in Figure 3. The TSI and the fiber-optic sensor demonstrated a maximum temperature difference around the tip of $0.5^{\circ} \mathrm{C}$. Fiber-optic sensor measurements were noisier than those for the TSI because of their lower $\left(0.8^{\circ} \mathrm{C}\right)$ precision compared with the TSI $\left(0.1^{\circ} \mathrm{C}\right)$. Furthermore, the average baseline temperature was 21.2 for fiber-optic sensor and 21.1 for TSI. The difference is within the accuracy of the measurements. The rise time of the TSI temperature was shorter than that for the fiber-optic sensor, most likely because of the finite distance $(<1 \mathrm{~mm})$ of the fiber-optic sensor to the electrode. The case temperature was measured as $21.5^{\circ} \mathrm{C}$ at the start of the MRI scan. At the 7th minute of scanning, the temperature was $21.8^{\circ} \mathrm{C}$. Electrode impedance was $700 \Omega$, and no significant change was recorded.

In the subacute experiment on the 14th day, when the animal was in the scanner under anesthesia, the case temperature was $36.3^{\circ} \mathrm{C}$ before starting the experiment. Additionally, the electrode impedance, which was $636 \Omega$ immediately after surgery, was measured as $498 \Omega$. The obtained anatomical image (MPRAGE) in implantfriendly mode is shown in Figure 4, and the temperature increase during this scan is shown in Figure 5. The

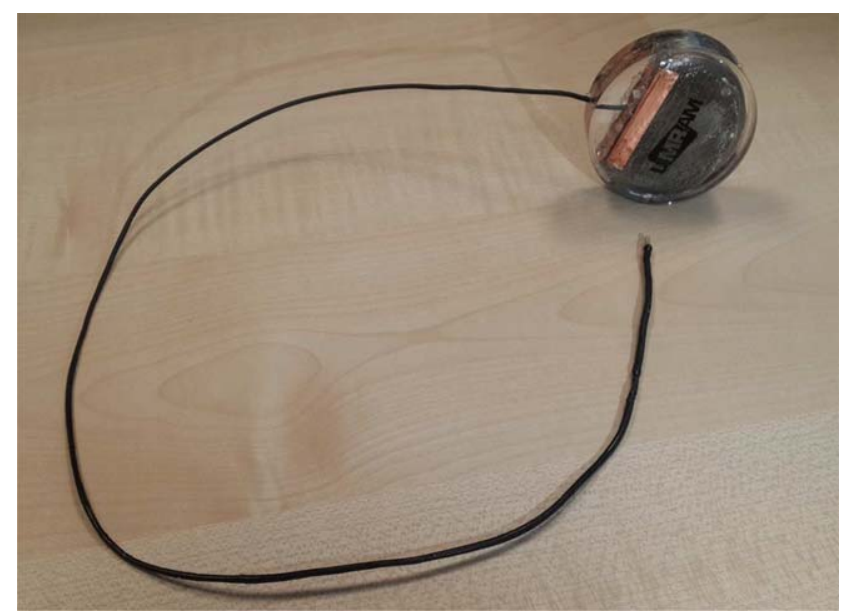

FIG. 2. Picture of the TSI device. The electronic circuit and the battery are inside a 50-mm-diameter titanium case with a 2-mmthick polydimethylsiloxane coating. A $60-\mathrm{cm}$-long lead with a diameter of $2.3 \mathrm{~mm}$ was connected to the case. The electrode at the tip of the lead has a $6 \mathrm{~mm}^{2}$ surface area. maximum temperature increase was $0.4^{\circ} \mathrm{C}$. As shown in Figure 6, the tip temperature before starting the experiment was $35.5^{\circ} \mathrm{C}$. After the MRI scans, the temperature increases around the electrode were measured as $12.8^{\circ} \mathrm{C}$ and $1.8^{\circ} \mathrm{C}$ for the maximum coupling and implantfriendly modes, respectively. For these scenarios, the temperature increases around the case were recorded as $2.4^{\circ} \mathrm{C}$ with the maximum coupling mode and $0.6^{\circ} \mathrm{C}$ with the implant-friendly mode. For the quadrature mode, the temperature increase was $4.1^{\circ} \mathrm{C}$ around the electrode and $1.2^{\circ} \mathrm{C}$ around the case. Finally, the electrode impedance values were measured as $498 \Omega, 557 \Omega$, and $488 \Omega$ before the scan, at the middle of the maximum coupling mode, and after the sequence, respectively. Similarly, for the implant-friendly mode, electrode impedance values were $491 \Omega, 507 \Omega$, and $491 \Omega$, respectively. For quadrature mode, the electrode impedance values were $496 \Omega, 526$ $\Omega$, and $495 \Omega$, respectively. There were temperaturedependent impedance changes in the measurements. A $10-\Omega$ difference was observed between the start and the end of the maximum coupling mode.

Histological examination was conducted on the brain of the animal, and gliosis and encephalomalacia were observed around the implant electrode as well as hemorrhage. Additionally, significant neovascularization, wall thickening, and vasodilatations were observed around the electrode.

\section{DISCUSSION}

We have presented a system for implant heating tests under MRI, and an application for the reduction of implant heating using dual-drive birdcage coil $(2,3)$ was demonstrated in vivo with a subacute animal experiment. An embedded thermistor inside the electrode tip provided temperature monitoring at the location most prone to heating.

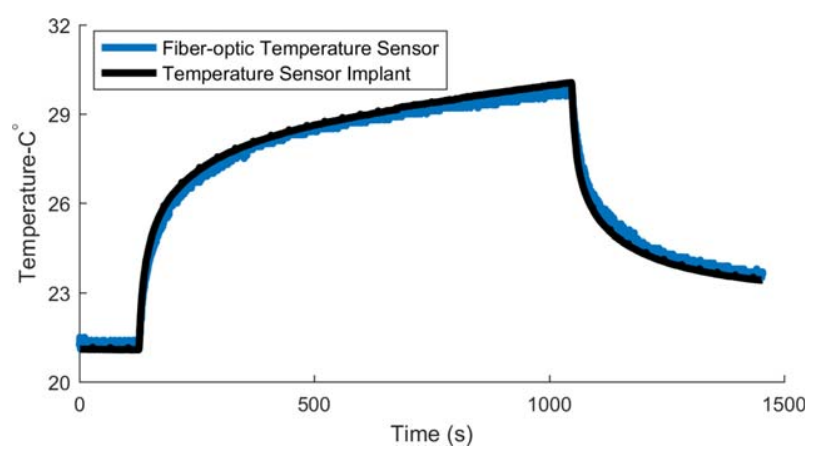

FIG. 3. Temperature change at the tip of the electrode. The black line is the measurement with the TSI, and the blue line is the measurement with the fiber-optic temperature sensor. 


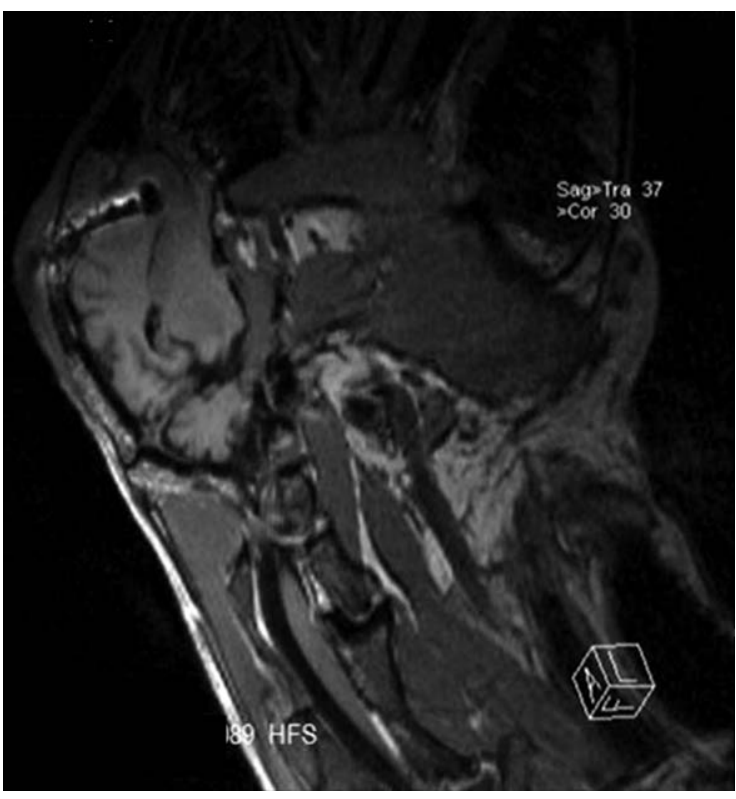

FIG. 4. MRI image of the lead placement obtained with 3DMPRAGE in implant-friendly mode after 2 wk of healing period.

\section{TSI Device}

The temperature measurement accuracy of the proposed device was verified using fiber-optic probes with phantom experiments. The $0.5^{\circ} \mathrm{C}$ maximum temperature difference at the tip confirmed the accuracy of the temperature measurement of the device during an MRI experiment. In addition, TSI temperature had a faster response that was probably a proximity effect of the measurement from the heating source. The TSI was measuring the source itself. On the contrary, a fiber-optic cable was placed as close as possible to the source. For the TSI, the measured temperature can be affected by the placement of the fiber-optic sensor. In the phantom experiment, the fiber-optic probe was attached at the closest position to the electrode, but it was still possible to suffer from the placement because it had a $400-\mu \mathrm{m}$ sensitive area. Similarly, TSI temperature measurement accuracy was affected by placement of the thermocouples. Hence, a thermocouple was directly placed inside the electrode, as they were in contact. The electrode was made of silver, which has high thermal conductivity $\left(427 \mathrm{~W} / \mathrm{mK}\right.$ at $\left.27^{\circ} \mathrm{C}\right)$; the temperature of the tissue was

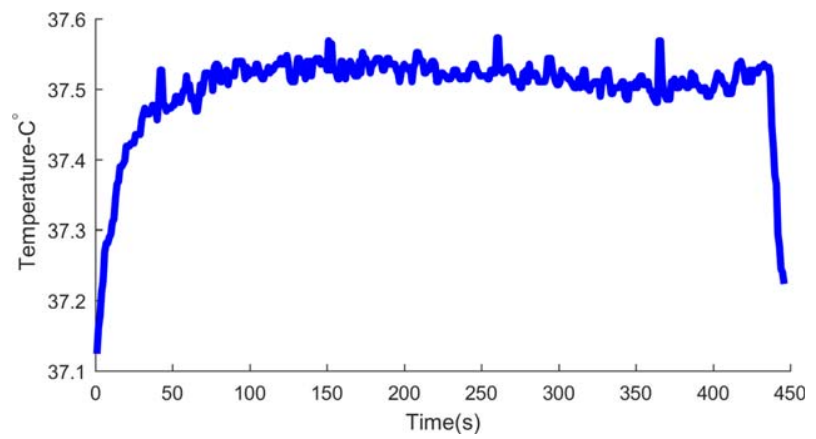

FIG. 5. Tip temperature rise during the anatomical imaging in implant-friendly mode.

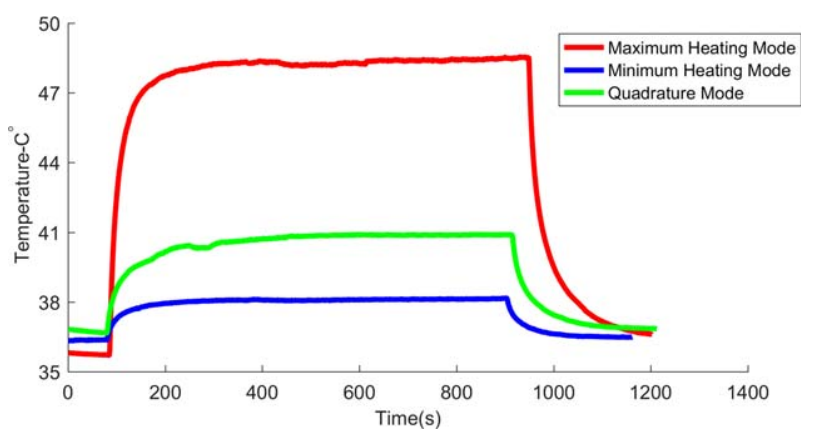

FIG. 6. Tip temperature measurements during the heating experiments. The temperature rise around the electrode is shown for the maximum coupling (red), implant-friendly (blue), and quadrature modes.

reflected directly to the thermocouple. This could explain why the TSI had a slightly faster response compared with the fiber-optic sensor. Above all, TSI temperature accuracy also depends on the battery value measurement of the device, the thermistor resistance tolerance, and the dynamic range of the ADC. The battery value can be correctly measured by the Bluetooth module, and no change was observed during the scan time. Because the battery voltage was updated in the software, the application used the updated supply voltages of the Wheatstone bridge supplies to calculate the temperature. Therefore, the battery voltage drop is not an issue as long as the device is operational. The resistance tolerance of the thermistor was $1 \%$, which corresponded to an accuracy of $0.3^{\circ} \mathrm{C}$ for our operating range and dominated the other error factors. The accuracy can be improved with some technological replacements if it is needed. A thermistor with a better resistance tolerance could decrease the error, but our error level was sufficient for this experiment.

The Bluetooth software was configured to achieve long battery life during the entire experimental procedure. For example, the sampling rate of the TSI was set to $1 \mathrm{~Hz}$, whereas the sampling rate of the fiber-optic temperature sensor was set to $5 \mathrm{~Hz}$. These settings were chosen to increase battery life, but also because the higher sampling rate was not providing additional information. Furthermore, the BLE connection parameters were a significant aspect of the power consumption. Therefore, the TSI and PC communication rates were adjusted to $1 \mathrm{~Hz}$. The default rate was higher than $25 \mathrm{~Hz}$. The rate of the communication was close to that of the temperature sampling rate. In addition, the TSI advertises itself every $10 \mathrm{~s}$. This was the slowest advertisement rate for the BLE device. All of these parameters were kept adjustable for the application flexibility. On the other hand, there can be environmental differences that may affect the power consumption; this was dependent on the surrounding Bluetooth devices when the device was not connected to the PC or the mobile device. If retransmissions are frequent due to the interference of other wireless devices, the power consumption can be higher. In addition, if the device received signal strength indication is very low during the experiments, the power consumption would increase. Although no significant battery 
drop was observed during the 2-wk experiment period, this could change according to the experimental conditions.

BLE technology was used for communication of the TSI with external devices. However, the majority of the implants use the Medical Device Radio Communications Service, which is regulated by the Federal Communications Commission, with a wireless communication frequency band of 401-457 MHz (27). This is problematic for our application because of the difficulty in eliminating the interference from high-power 123-MHz RF signals inside the 3T MRI scanner with a simple filter. Therefore, the BLE frequency band of $2402-2480 \mathrm{MHz}$ is more suitable for wireless communication during real-time monitoring. Bluetooth communication also has disadvantages. In the 2-GHz range, skin depth dramatically limits the range of communication. This may explain the observed maximum of $2 \mathrm{~m}$ communication distance for our device.

It should be noted that the proposed design is not intended as a modification of clinical AIMDs but rather a tool for animal testing of these implants in subacute or chronic conditions. To incorporate the proposed design to a clinical AIMD device, extensive investigation has to be done.

We used the implant lead as the Bluetooth antenna; however, a designated antenna for the Bluetooth communication can be implemented on the TSI to increase the range. For future MRI compatible implants, it is possible to implement such wireless communication circuitries for data monitoring during MRI scan. A recent frequency band, the Medical Body Area Network, is regulated in the 2360-2400 MHz band for data collection for a short distance from medical devices (27). For future work, the implant can also be configured for different monitoring purposes. Because it has Bluetooth smart capabilities, it can sleep and save power most of the time, but at certain time intervals it can wake up and collect data regarding the case temperature and impedance from its sensors as an Internet of Things device. Then, the device can send the data to computers and an Internet network for patient safety monitoring.

This study has several limitations. The shape and dimension of the TSI were not chosen to mimic a specific AIMD, because our goal was to introduce a new in vivo method of testing rather than test a commercial AIMD. There are many different sizes and shapes of AIMDs available in the market. To test a specific implant, the TSI design can be used in place of the electronic circuit of the implant, and in vivo testing can be conducted. Our plan, however, is to obtain a generic comparison between chronic, subacute animal testing and phantom testing with this capability in the future. Another limitation of this study was extension of the USB cable without considering RF leakage to the room. This decreased the image quality, but in our experiments this was acceptable because imaging was not the main target. In the future, we are planning to solve this issue by placing a filter.

\section{Animal Experiments}

Before the heating experiments, there was a 2-wk recovery period. Although this period is too short for complete healing of the brain tissue, it is sufficient to eliminate most of the acute effects of the implant surgery. Healing of the subcutaneous wounds and the disappearance of edema around the TSI case was sufficient for demonstration of the device performance and the in vivo heating reduction experiments. In addition, subacute experiments were sufficient to demonstrate that the TSI enables the collection of experimental data without further surgical operation. The long battery life and usage of medical grade materials imply that TSI can be used for subacute and chronic analysis of heating of AIMDs. Because this study focused on showing the capability of TSI by minimizing the invasive nature of heating experiments, subacute experiments were sufficient, thus eliminating unnecessary discomfort to the animal. Future studies can focus on longer tissue recovery processes.

A comparison of the temperature curves provided in Figure 3 (in vitro) and Figure 6 (in vivo) shows that the in vivo temperature was achieved at steady state, whereas the in vitro temperature curve had a positive slope. This behavior could be due to the cooling effect of the perfusion in the tissue. The effect of the perfusion and potential overestimation of in vitro studies can be analyzed by comparing in vivo experiments in living and sacrificed animals. In addition, TSI could be used in other parts of the body to understand the perfusion effect in different regions.

Subacute in vivo heating experiments were extended to show an application of an existing implant heating reduction technique $(2,3)$. The performance of the heating reduction can be affected from different scan parameters; however, our intent was not to examine the performance of the technique. Here, three heating experiment results have been shown by only changing the phase and amplitude applied to each of the channels of the dual-drive birdcage coil. Extreme heating can be observed, but it can be reduced to considerable levels for patient safety. Moreover, parallel transmission with more channels can be another solution for MRI implant heating reduction. There are promising studies that show the reduction of the tip heating by parallel transmission techniques (5-7). Future works can be extensively concentrated on parallel transmission techniques and its clinical use for reduction of implant heating.

The histology provided interesting results about damage around the electrode. However, histology was conducted just after the MRI examination and 15 days after the surgery; therefore, it was not possible to differentiate the MRI-related heating and the surgery effects. To provide a definite conclusion, the time between the MRI examination and the surgery should be increased further and tested on multiple animals with controls. The aim of our study, however, was to introduce the TSI. Here, we showed the capability of TSI with a sample study.

We measured the electrode impedance before, during, and after the heating experiment. Our expectation was to observe impedance change due to the heating-related tissue damage. For this study, we observed an electrode impedance increase when there was an increase in the tip temperature. Furthermore, after the sequence, the value of the electrode impedance dropped back to its starting value except the slight change after the 
maximum coupling mode. At this time, these results indicate that the TSI can improve our understanding of in vivo mechanisms during temperature changes in the tissue. Although there was a drop in electrode impedance between the acute and subacute experiments, the measured electrode impedance falls into clinical measurements with a theoretical analysis that are 500-1500 $\Omega$ (28). We cannot conclude what caused the drop in impedance between acute and subacute experiments and impedance changes related to the temperature changes. These effects should be examined rigorously with controlled experiments on multiple animals.

\section{CONCLUSION}

In this study, a TSI was designed for use in MRI heating experiments to reduce acute surgical effects. Temperature measuring accuracy of the TSI was comparable to the fiber-optic temperature sensors, with a maximum error of $0.5^{\circ} \mathrm{C}$. Furthermore, in vivo subacute animal experiments were shown while steering the E-field using a dual-drive birdcage coil. By applying similar whole body-averaged SAR values, the changes in electrode temperature were recorded as $1.8^{\circ} \mathrm{C}, 12.8^{\circ} \mathrm{C}$, and $4.1^{\circ} \mathrm{C}$ and the changes in case temperature were $0.6^{\circ} \mathrm{C}, 2.4^{\circ} \mathrm{C}$, and $1.2^{\circ} \mathrm{C}$ in the implant-friendly, maximum coupling, and quadrature modes, respectively. In summary, our proposed TSI implant enables more accurate in vivo heating experiments.

\section{REFERENCES}

1. Nyenhuis JA, Sung-Min P, Kamondetdacha R, Amjad A, Shellock FG, Rezai AR. MRI and implanted medical devices: basic interactions with an emphasis on heating. IEEE Trans Dev Mater Reliab 2005;5: 467-480.

2. Eryaman Y, Turk EA, Oto C, Algin O, Atalar E. Reduction of the radiofrequency heating of metallic devices using a dual-drive birdcage coil. Magn Reson Med 2013;69:845-852.

3. Eryaman Y, Akin B, Atalar E. Reduction of implant RF heating through modification of transmit coil electric field. Magn Reson Med 2011;65:1305-1313.

4. Griffin GH, Anderson KJT, Celik H, Wright GA. Safely assessing radiofrequency heating potential of conductive devices using imagebased current measurements. Magn Reson Med 2015;73:427-441.

5. Eryaman Y, Guerin B, Akgun C, et al. Parallel transmit pulse design for patients with deep brain stimulation implants. Magn Reson Med 2015;73:1896-1903.

6. Gudino N, Sonmez M, Yao Z, et al. Parallel transmit excitation at 1.5 $\mathrm{T}$ based on the minimization of a driving function for device heating. Med Phys 2015;42:359-371.

7. Etezadi-Amoli M, Stang P, Kerr A, Pauly J, Scott G. Controlling radiofrequency-induced currents in guidewires using parallel transmit. Magn Reson Med 2015;74:1790-1802.

8. Golestanirad L, Iacono MI, Keil B, et al. Construction and modeling of a reconfigurable MRI coil for lowering SAR in patients with deep brain stimulation implants. Neuroimage 2016;147:577-588.

9. Murbach M, Zastrow E, Neufeld E, Cabot E, Kainz W, Kuster N. Heating and safety concerns of the radio-frequency field in MRI. Curr Radiol Rep 2015;3:45.
10. Mattei E, Triventi M, Calcagnini G, Censi F, Kainz W, Mendoza G, Bassen HI, Bartolini P. Complexity of MRI induced heating on metallic leads: experimental measurements of 374 configurations. Biomed Eng Online 2008;7:11.

11. Rezai AR, Finelli D, Nyenhuis JA, Hrdlicka G, Tkach J, Sharan A, Rugieri P, Stypulkowski PH, Shellock FG. Neurostimulation systems for deep brain stimulation: in vitro evaluation of magnetic resonance imaging-related heating at 1.5 Tesla. J Magn Reson Imaging 2002;15: 241-250.

12. Nordbeck P, Weiss I, Ehses P, et al. Measuring RF-induced currents inside implants: impact of device configuration on MRI safety of cardiac pacemaker leads. Magn Reson Med 2009;61:570-578.

13. Georgi JC, Stippich C, Tronnier VM, Heiland S. Active deep brain stimulation during MRI: a feasibility study. Magn Reson Med 2004; 51:380-388.

14. Carmichael DW, Pinto S, Limousin-Dowsey P, Thobois S, Allen PJ, Lemieux L, Yousry T, Thornton JS. Functional MRI with active, fully implanted, deep brain stimulation systems: safety and experimental confounds. Neuroimage 2007;37:508-517.

15. Shellock FG, Fieno DS, Thomson LJ, Talavage TM, Berman DS. Cardiac pacemaker: in vitro assessment at 1.5 T. Am Heart J 2006;151: 436-443.

16. Kahan J, Papadaki A, White M, Mancini L, Yousry T, Zrinzo L, Limousin P, Hariz M, Foltynie T, Thornton J. The safety of using body-transmit MRI in patients with implanted deep brain stimulation devices. Plos One 2015;10:e0129077.

17. Yeung CJ, Susil RC, Atalar E. RF safety of wires in interventional MRI: using a safety index. Magn Reson Med 2002;47:187-193.

18. Acikel V, Atalar E. Modeling of radio-frequency induced currents on lead wires during MR imaging using a modified transmission line method. Med Phys 2011;38:6623-6632.

19. Acikel V, Uslubas A, Atalar E. Modeling of electrodes and implantable pulse generator cases for the analysis of implant tip heating under MR imaging. Med Phys 2015;42:3922-3931.

20. Park SM, Kamondetdacha R, Nyenhuis JA. Calculation of MRIinduced heating of an implanted medical lead wire with an electric field transfer function. J Magn Reson Imaging 2007;26:1278-1285.

21. Smith CD, Kildishev AV, Nyenhuis JA, Foster KS, Bourland JD. Interactions of magnetic resonance imaging radio frequency magnetic fields with elongated medical implants. J Appl Phys 2000;87:61886190.

22. Feng S, Qiang R, Kainz W, Chen J. A technique to evaluate MRIinduced electric fields at the ends of practical implanted lead. IEEE T Microw Theory 2015;63:305-313.

23. Akca IB, Ferhanoglu O, Yeung CJ, Guney S, Tasci TO, Atalar E. Measuring local RF heating in MRI: simulating perfusion in a perfusionless phantom. J Magn Reson Imaging 2007;26:1228-1235.

24. Gorny KR, Presti MF, Goerss SJ, et al. Measurements of RF heating during 3.0-T MRI of a pig implanted with deep brain stimulator. Magn Reson Imaging 2013;31:783-788.

25. Luechinger R, Zeijlemaker VA, Pedersen EM, Mortensen P, Falk E, Duru F, Candinas R, Boesiger P. In vivo heating of pacemaker leads during magnetic resonance imaging. Eur Heart J 2005;26:376-383.

26. Bluetooth Technology Website. Technical considerations. https:// www.bluetooth.com/specifications/bluetooth-core-specification/technicalconsiderations. Accessed April 13, 2017.

27. Bluetooth Technology Website. Proximity and RSSI. https://blog.bluetooth.com/proximity-and-rssi. Accessed April 13, 2017.

28. Federal Communications Commission. Medical Device Radiocommunications Service (MedRadio). https://www.fcc.gov/general/medicaldevice-radiocommunications-service-medradio. Published June 14, 2016. Accessed November 1, 2016.

29. Butson CR, Maks CB, Mcintyre CC. Sources and effects of electrode impedance during deep brain stimulation. Clin Neurophysiol 2006; 117:447-454. 